\title{
CARs: new models abound
}

\begin{tabular}{|c|c|}
\hline $\begin{array}{l}\text { this system } \\
\text { may also be } \\
\text { adaptable to } \\
\text { the treatment } \\
\text { of solid } \\
\text { tumours, } \\
\text { as well as } \\
\text { indications } \\
\text { beyond cancer } \\
\text { therapy }\end{array}$ & $\begin{array}{l}\text { Adoptive transfer of T cells } \\
\text { transduced with chimeric antigen } \\
\text { receptors (CARs) has emerged as a } \\
\text { powerful therapy for B cell malignan- } \\
\text { cies. However, current CAR T cell } \\
\text { treatments are limited by safety con- } \\
\text { cerns, and their utility for other types } \\
\text { of cancer is restricted by the rarity of } \\
\text { unique cancer antigens. Now, three } \\
\text { studies show how the utility of CAR } \\
\text { T cells might be expanded. Reporting } \\
\text { in Proceedings of the National } \\
\text { Academy of Sciences USA, two papers } \\
\text { by the groups of Schultz, Young, Kim } \\
\text { and co-workers present switchable } \\
\text { CAR (sCAR) T cells, which can be } \\
\text { rapidly activated or re-directed to } \\
\text { different antigens. And reporting in } \\
\text { Cell, Lim and colleagues present CAR } \\
\text { T cells that are only activated by } \\
\text { particular combinations of anti- } \\
\text { gens - thereby allowing for precise } \\
\text { targeting of tumour cells even in the } \\
\text { absence of unique cancer antigens. }\end{array}$ \\
\hline
\end{tabular}

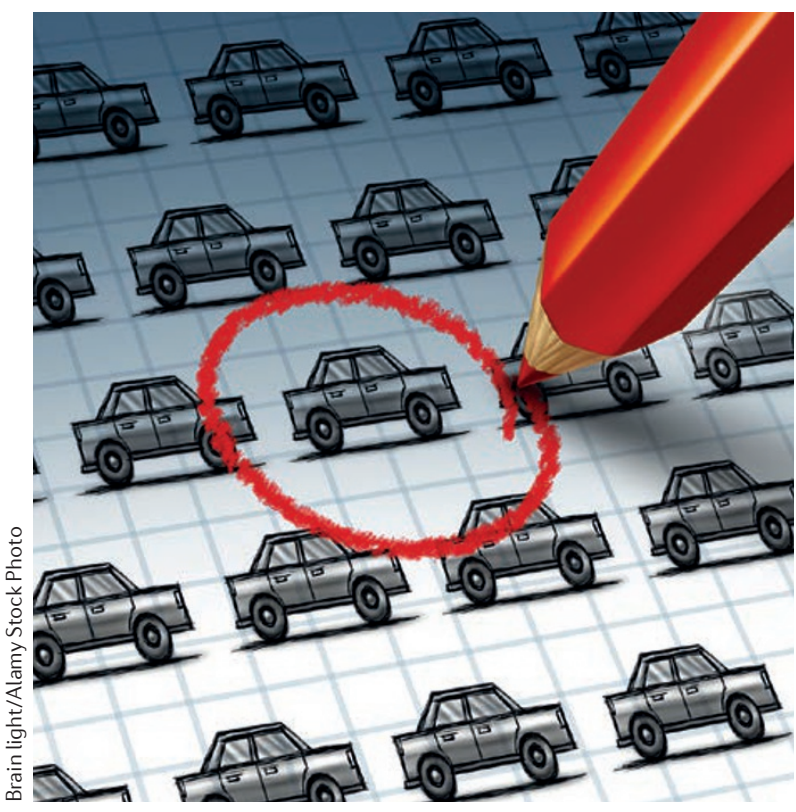

CARs are composed of an extracellular single-chain antibody variable domain that is fused to intracellular co-stimulatory and activation domains. So far, the greatest successes of CAR T cells have been achieved in targeting the pan-B cell antigen CD19 in B cell acute lymphoblastic leukaemia (B-ALL). However, patients are left permanently depleted of B cells, and the cancer can reappear owing to antigen-loss escape mutants. Moreover, once CAR T cells are unleashed into the bloodstream they are hard to control and can induce fatal complications. CARs with 'kill switches' have been engineered; however, these do not allow control over cell activation and lead to the irreversible elimination of the CAR T cells. By contrast, the new sCARs bind to a specific peptide neo-epitope, which can be engineered into antibodies, or to fluorescein, which can be coupled to antibodies. Consequently, sCAR T cells only become activated in the presence of engineered cancer-specific antibodies ('switches') that carry the particular modifications - combining the versatility of antibody-based approaches with the efficacy of CAR T cells.

Using mouse models of B-ALL, Schultz, Young, Kim and co-workers demonstrated that sCAR T cells, in combination with a CD19-directed switch, were just as efficient in eliminating B cells as conventional CD19-specific CAR T cells. However, the activity of sCAR T cells could be titrated, as it is strictly dependent on the presence of the switch, and when the switch-treatment was stopped in a surrogate immunocompetent mouse model, B cells rebounded. Moreover, the specificity of the sCAR T cells was readily switchable to other $\mathrm{B}$ cell antigens such as CD20 or CD22, a strategy that could be useful to catch escape mutants. The authors argue that this system may also be adaptable to the treatment of solid tumours, as well as indications beyond cancer therapy.

By contrast, Lim and colleagues sought to limit potential toxicity and overcome constraints that are due to the rarity of unique cancer antigens by engineering combinatorial antigen-sensing circuits. They engineered a synthetic Notch (synNotch) receptor, consisting of an extracellular antibody-recognition domain fused to a transcriptional activator domain, which can induce the expression of a CAR construct. To provide proof of principle for dual antigen targeting, the antigen-binding domain of synNotch was directed against green fluorescent protein (GFP) and the CAR was targeted at CD19. The resulting SynNotch CAR T cells were then injected into mice that had previously been engrafted with GFP- and CD19-positive B-ALL cancer cells in one flank and with their GFPnegative counterparts in the other flank. The synNotch-CAR T cells only killed cancer cells that expressed both antigens. The authors propose that this system may be adapted to any combination of antigens and thereby allow the targeting of a wide range of different tumours.

Alexandra Flemming

ORIGINAL ARTICLES Rodgers, D. T. et al. Switchmediated activation and retargeting of CAR-T cells for B-cell malignancies. Proc. Natl. Acad. Sci. USA 113, E459-E468 (2016) | Ma, J. S. Y. et al. Versatile strategy for controlling the specificity and activity of engineered T cells. Proc. Natl. Acad. Sci. USA 113, E450-E458 (2016)|Roybal K. T. et al. Precision tumour recognition by $T$ cells with combinatorial antigen-sensing circuits. Cell 164, 770-779 (2016) 